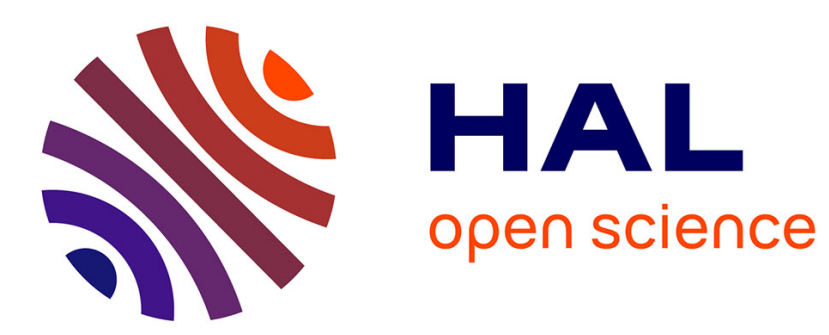

\title{
Competitive Location in Cognitive Radio Networks
}

Stefano Iellamo, Ekaterina Alekseeva, Lin Chen, Marceau Coupechoux, Yuri

\author{
A. Kochetov
}

\section{To cite this version:}

Stefano Iellamo, Ekaterina Alekseeva, Lin Chen, Marceau Coupechoux, Yuri A. Kochetov. Competitive Location in Cognitive Radio Networks. 4OR: A Quarterly Journal of Operations Research, 2015, 13 (1), pp.81-110. 10.1007/s10288-014-0268-1 . hal-01144293

\section{HAL Id: hal-01144293 https://hal-imt.archives-ouvertes.fr/hal-01144293}

Submitted on 14 Oct 2019

HAL is a multi-disciplinary open access archive for the deposit and dissemination of scientific research documents, whether they are published or not. The documents may come from teaching and research institutions in France or abroad, or from public or private research centers.
L'archive ouverte pluridisciplinaire HAL, est destinée au dépôt et à la diffusion de documents scientifiques de niveau recherche, publiés ou non, émanant des établissements d'enseignement et de recherche français ou étrangers, des laboratoires publics ou privés. 


\title{
Competitive location in cognitive radio networks
}

\author{
Stefano Iellamo • Ekaterina Alekseeva • \\ Lin Chen • Marceau Coupechoux • Yuri \\ Kochetov
}

Received: date / Accepted: date

\begin{abstract}
The paper addresses the problem of strategic base stations placement in cognitive radio networks. We consider a primary user, operating on the frequency channels of a primary network, and an operator (a leader) facing the competition of a second operator (a follower). These operators are willing to exploit the unused capacity of the primary network and maximize their profits derived from operating the base stations installed and clients served. The leader is aware of the future arrival of the follower, who is able to capture clients by placing its own base stations. It has also to limit the interference power at some measurement points defined by the primary user. We formulate the problem as a bi-level location problem and develop a matheuristic where a mixed integer program derived from the follower's problem is solved by CPLEX software. We prove that the follower's problem is NP-hard and the leader's problem is $\Sigma_{2}^{P}$-hard. Our computational experiments confirm the value of competition for the strategic planning in cognitive radio networks.
\end{abstract}

Keywords Bi-level programming - Metaheuristics · Competitive location · Leader-follower game $\cdot$ Cognitive radio networks

The research of the second and the fifth authors was partially supported by RFBR grants 12-01-00077 and 13-07-00016.

S. Iellamo, M. Coupechoux

Telecom ParisTech, LTCI CNRS 5141, 75013 Paris, France

E-mail: iellamo@enst.fr, coupecho@enst.fr

E. Alekseeva, Y. Kochetov

Novosibirsk State University, Sobolev Institute of Mathematics

4 Acad. Koptyuga ave., 630090 Novosibirsk, Russia

E-mail: ekaterina2@math.nsc.ru, jkochet@math.nsc.ru

L. Chen

University of Paris-Sud XI, Laboratoire LRI, 91405 Orsay, France

E-mail: chen@lri.fr 


\section{Introduction}

Cognitive radio (CR) whose core idea is opportunistic spectrum access enables unlicensed users to opportunistically access under-utilized legacy spectrum bands. The possibility of accessing the existing white spaces of the legacy spectrum (e.g., TV band) opens the door to wireless service providers (WSPs) for new commercial opportunities. In the perspective of such a new paradigm bringing new opportunities and challenges, the following natural while crucial questions deserve an in-depth analysis. What is the optimal deployment strategy for a WSP to maximize its profit while not violating the constraint imposed by a primary user (PU)? How is the white space spectrum partitioned among multiple WSPs that enter the market in different times? What is the gain for a WSP of anticipating the arrival of a competitor?

In this paper we look at the base station location problem from a game theory perspective. We study the deployment of stations in the vicinity of a primary network by competitive WSPs. We consider a primary network operating on a licensed frequency band made of several frequency channels. Two competitive CR operators, a leader and a follower, getting to a market sequentially, are willing to deploy a secondary network by opportunistically exploiting the unused downlink capacity of the primary network for their downlink transmissions. Each operator can place a set of stations at potential sites and set their corresponding transmission powers so as to cover the maximum number of clients.

The leader allowed deploying its network should satisfy a number of constraints. First, it has a budget constraint which limits the possible number of stations to be installed. Then, it has to make sure that the deployment of the secondary network does not impair the quality of the service of the primary receivers. To this end, there are the measurement points (MP), possibly proposed by the PU, at which the power received from the secondary network must be controlled under a certain interference temperature threshold. At last, the leader has to take into account the future arrival of the follower who is able to capture some clients by appropriately placing its own stations to maximize its total profit.

This problem belongs to the class of the well-studied competitive facility location problems in discrete combinatorial optimization [2]. In a competitive problem there is a two level hierarchy with one decision maker (or a player) at each level. The highest level player optimizes its objective function taking into account the intention of the lower level player to optimize its own objective. The problem might be written as a mixed integer bi-level programming model. The bi-level nature of the problem forces to explore two cases of the follower's behavior: cooperative and non-cooperative. We present a new bi-level mathematical programming model for the problem considered and establish the complexity status of the problem. We propose a matheuristical approach to find approximate solutions to the problem. Matheuristics that generally are hybrids of any heuristics (simulating annealing, tabu search or genetic algorithms) and exact methods (branch-and-bound, branch-and-cut) are widely 


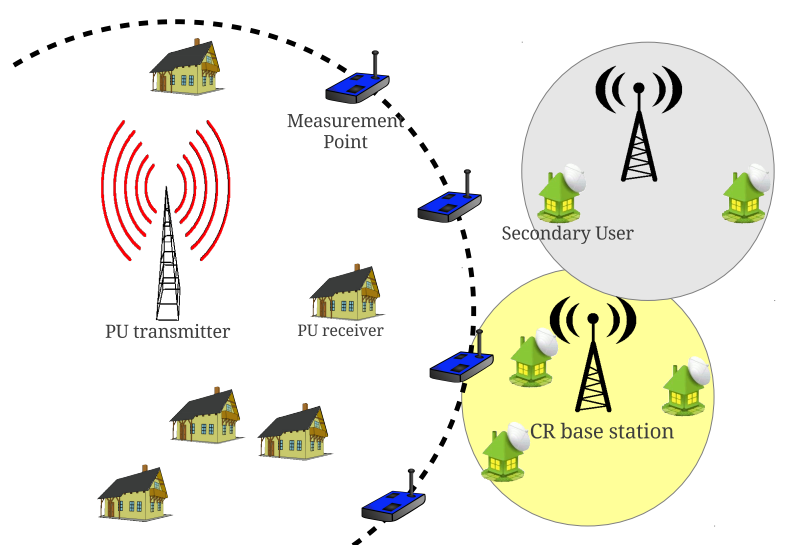

Fig. 1 Network Model: two CR operators serve clients

used in operations research. Originally they were developed for single-level problems to get the solutions of high quality. Here we have adopted one of them for the bi-level case.

From the application point of view the network model considered might be applicable in some typical CR scenarios. One of these is the deployment of IEEE 802.22 networks in the UHF/VHF bands. The primary network is a set of Digital Television (DTV) antennas broadcasting TV programs on one or several frequency channels. The secondary network is a IEEE 802.22 network offering web services to various customers like individuals, small size companies or schools. In order to protect TV receivers, the concept of keep-out region has been proposed [30]. Within this area clients are not allowed emitting in the associated DTV channels. Practically, a set of MPs can be defined on the contour of the keep-out zone or on specific points within the zone, and the interference measured at these points should be controlled under a certain threshold.

Figure 1 illustrates the network model considered. In this example the PU's transmitter uses a channel and is characterized by a keep-out zone (delimited by the dashed line). Such an area might be calculated using propagation models, transmitters characteristics and interference requirements at receivers [30]. In this example the PU has defined some MPs on the border of the keep-out zone. The stations placed outside the zone are allowed serving the clients using the channel provided that the interference received at MPs is below a threshold defined by the PU.

The paper is organized as follows. In Section 2 we present some related works. We give all the necessary notations and definitions in Section 3. Then we formulate the competitive station location problem using mixed integer linear bi-level programming in Section 4. We distinguish two sub-cases depending 
on whether the follower behaves in cooperative or non-cooperative manner in Section 4.1. Thus we can derive upper and lower bounds to the leader's profit, respectively, Section 4.2. We show that the leader's problem is $\Sigma_{2}^{P}$-hard and that the follower's problem is NP-hard in the strong sense in Section 5. We propose a matheuristic based on probabilistic tabu search (TS) and mathematical programming in order to find the approximate solutions to the bi-level problem in Section 6. We discuss the consistency of our generic model with respect to the IEEE 802.22 standard and some directions for the future work in Section 7. We highlight through the numerical results the importance for an operator to anticipate competition in Section 8. We end with the conclusion in Section 9 and a list of literature.

\section{Related works}

The station location problem has been well investigated in the literature. In [1] authors formulate the station location problem in a CDMA network using mixed integer programming model and tabu search algorithm to obtain approximate solutions for locations of stations and their configurations. The competitive model assumed in our work is however absent from this literature since mobile operators have their own spectrum.

Several recent works have studied base station location games $[4,25]$ where a Stackelberg game is formulated that combines the location of stations and mobile association problems. These papers give interesting insights of the problem on a line network. However they can hardly be used for practical network planning. In [26] competition between CR networks is considered from a point of view of an auction mechanism between TV broadcasters and WSPs. Competition for the potential locations is not taken into account.

Quantifications of the white space available spectrum are presented in [12] and [27]. In [11] authors present a thorough analysis of DTV white spaces including their non-homogeneous nature in terms of available channel bandwidth and propagation characteristics. Several works tackle the resource allocation problem in IEEE 802.22 networks [16].

A traditional way of avoiding the interference of stations on DTV receivers is to define a keep-out region around the DTV transmitter where stations cannot be placed. Only the effect of the closest one is usually considered in the derivation of this region. However in [30] the authors show how cumulative interference can modify the keep-out region. Few works consider the effect of cumulative interference in IEEE 802.22 networks. In [32] a method for evaluating the effect of CR stations on DTV coverage and on the inter-system isolation distance is proposed. Stations are supposed to form a traditional hexagonal network and the location problem is not raised.

The competitive facility location problem is extensively studied in economics. The leader is a firm willing to open facilities at some potential sites and tries to anticipate the arrival of a competitor [20]. The problem is formulated using bi-level programming. The effects of resource allocation (channels 
and power) and cumulative interference make however the CR base station location problem completely new with respect to this literature.

Mixed integer linear bi-level programs are known to be intrinsically hard to solve. Traditional approaches for solving such programs involve either vertex enumeration $[10,14]$ or replace the follower's problem by the Karush-KuhnTucker optimality conditions in order to boil down to a single level optimization problem [5]. The need for good near optimal solutions for large size problems obtained in a reasonable time has given the rise to metaheuristics for solving bi-level programs [21]. In this paper we propose a hybrid algorithm (or matheuristics [7]) based on tabu search and mathematical programming similar to one presented in [3].

\section{Notations and problem statement}

We consider two competitive operators which we refer to as a leader and a follower due to their sequential entering a market where a primary network has already existed. They compete to serve clients by installing and configuring own CR networks. The leader makes a decision first taking into account the arrival of the follower.

Let $\mathcal{N}$ be a finite set of clients. We assume that they are equipped with directional or omni-directional antennas for the signal reception. Each client $j \in \mathcal{N}$ is characterized by a positive weight $w_{j}$ which stands for the expected benefit that can be derived by the operator from serving this client. These weights can be used to classify clients into different categories, each of which is representative of a certain class of payment. Typical examples of such categories might be "individual customer", "company", or "school".

Let $\mathcal{S}$ be a finite set of identified potential sites where the operators can install their stations. Each site can host at most one station. For short, we will further write that an operator installs station $i$ that means installing a station at site $i$ from $\mathcal{S}$. The unit operational cost of each station is $\lambda$. Hence, installing a station is convenient only if the clients served guarantee a revenue greater than $\lambda$. We suppose that the leader has a finite initial budget permitting to build at most $K$ stations. On the contrary, we suppose that the follower is able to afford any initial expenses. This makes sense because it allows us to find the leader's perspective for the worst case planning assumptions.

Let $\mathcal{T}$ be a finite set of PU transmitters (e.g. TV transmitters). Each PU transmitter operates on a subset of set $\mathcal{C}$ of frequency channels. The operators can reuse the channels licensed to the PU.

To configure a station at site $i \in \mathcal{S}$ each operator must choose the operational frequency channel $c \in \mathcal{C}$ and tune its transmit power which cannot exceed the maximum allowed transmit power $\bar{P}$. We assume that each station can use no more than one frequency channel. Bonding or aggregation of channels is not supported.

In order to protect primary network receivers (e.g. DTV receivers) we assume that the PU has defined finite set $\mathcal{M}$ of MPs which intend to control 
the interference temperature. The interference temperature at each $m \in \mathcal{M}$ on each channel $c \in \mathcal{C}$ is defined as

$$
\sum_{i \in \mathcal{S}} h_{i m c} p_{i c}
$$

where $h_{i m c}$ is the channel gain on frequency $c$ between station $i$ and MP $m, p_{i c}$ is the transmit power defined by the operator (in this term by the leader). We assume that the interference temperature at each MP $m$ on each channel $c$ cannot exceed some pre-defined threshold value $\bar{I}_{m c}$. The network designer faces the trade-off of protecting PU receivers while maintaining computational efforts at a reasonable level. Different approaches can be considered. For instance, MPs can be placed: (a) using a grid of tightly spaced points; (b) at strategic locations (e.g. isolated facilities); (c) along roads; (d) or on the keep-out region boundary. The threshold value $\bar{I}_{m c}$ can also be increased to protect a region around a MP.

We assume that client $j$ might be covered by station $i$ on channel $c$ if its Signal to Interference plus Noise Ratio (SINR) is above a specified threshold $\bar{\gamma}$. The SINR of client $j$ is defined as:

$$
\frac{g_{i j c}^{i} p_{i c}}{\sum_{\substack{i^{\prime} \in \mathcal{S} \\ i^{\prime} \neq i}} g_{i^{\prime} j c}^{i} p_{i^{\prime} c}+\sum_{k \in \mathcal{T}} h_{k c} l_{k j c}^{i}+N_{c}}
$$

where $g_{i j c}^{i}\left(g_{i^{\prime} j c}^{i}\right)$ and $l_{k j c}^{i}$ are the channel gains on frequency $c$ between station $i\left(i^{\prime}\right)$ and client $j$ and between the PU's transmitter $k$ and client $j$, respectively, the superscript $i$ indicates that the client's antenna is oriented towards station $i$; $h_{k c}$ is the transmit power of PU $k$ on channel $c ; N_{c}$ is the background thermal noise on the channel $c$. Further the absence of a superscript in $g_{i j c}$ means that client $j$ uses an omni-directional antenna. The channel gains between two geographical points can be estimated by using prediction tools (e.g. HataOkumura model, statistical models or ray tracing) or obtained by on-field measurements. They are assumed to be known to the CR operators.

We assume that client $j$ might be served by station $i$ if the SINR on the chosen frequency $c$ between station $i$ and client $j$ is greater then threshold $\bar{\gamma}$ and $i$ is the station providing the strongest signal power received. This model assumes that every client is able to do isotropic signal measurements ${ }^{1}$. If the leader and the follower provide client $j$ with the same strongest signal power from different channels, then the follower channel is preferred. It is the most pessimistic scenario for the leader to estimate the worst case of total profit. Note that another behavior of clients is assumed in the most part of the competitive location models $[2,6,17-19]$.

1 It means that every client has either a rotating directional antenna as assumed in [16] or a second omni-directional antenna used for measurements. 


\section{Mathematical model}

As mentioned in the introduction, we are interested in the problem of strategic base station placement where two providers enter the market at different times (a leader and a follower), deploy their stations on possible candidate sites and set their transmission powers so as to maximize their profits. In this section, we model this problem as a bi-level optimization problem and provide the formal analysis of its complexity.

Let us introduce two groups of the decision variables. The first group is the leader's variables:

$$
\begin{aligned}
x_{i c} & =\left\{\begin{array}{l}
1 \text { if the leader installs station } i \text { and operates on channel } c, \\
0, \text { otherwise, }
\end{array}\right. \\
x_{i j c} & = \begin{cases}1 \text { if client } j \text { is served by the leader's station } i \text { on channel } c, \\
0, \text { otherwise, }\end{cases}
\end{aligned}
$$

and non-negative variables $p_{i c}$ which mean the transmit power from station $i$ on channel $c$ for each $i \in \mathcal{S}, c \in \mathcal{C}$, and client $j \in \mathcal{N}$. Denote $x=\left\{x_{i c}\right\}_{i \in \mathcal{S}, c \in \mathcal{C}}$, $X=\left\{x_{i j c}\right\}_{i \in \mathcal{S}, j \in \mathcal{N}, c \in \mathcal{C}}$, and $p=\left\{p_{i c}\right\}_{i \in \mathcal{S}, c \in \mathcal{C}}$, for short.

The second group is the follower's variables: the binary decision variables $y_{i c}, y_{i j c}$, and non-negative variables $q_{i c}$ for each $i \in \mathcal{S}, c \in \mathcal{C}$, and $j \in \mathcal{N}$ with the similar meanings but for the follower. Denote $y=\left\{y_{i c}\right\}_{i \in \mathcal{S}, c \in \mathcal{C}}, Y=$ $\left\{y_{i j c}\right\}_{i \in \mathcal{S}, j \in \mathcal{N}, c \in \mathcal{C}}$, and $q=\left\{q_{i c}\right\}_{i \in \mathcal{S}, c \in \mathcal{C}}$, for short.

Now the competitive station location problem can be written as a following bi-level mixed integer linear programming model:

$$
\max _{x, X, p}\left(\sum_{j \in \mathcal{N}} w_{j} \sum_{i \in \mathcal{S}} \sum_{c \in \mathcal{C}} x_{i j c}-\lambda \sum_{i \in \mathcal{S}} \sum_{c \in \mathcal{C}} x_{i c}\right)
$$

subject to

$$
\begin{aligned}
& \sum_{i \in \mathcal{S}} \sum_{c \in \mathcal{C}}\left(x_{i j c}+y_{i j c}^{*}\right) \leq 1 \forall j \in \mathcal{N} \\
& \sum_{i \in \mathcal{S}} \sum_{c \in \mathcal{C}} x_{i c} \leq K \\
& \sum_{c \in \mathcal{C}} x_{i c} \leq 1 \quad \forall i \in \mathcal{S} \\
& p_{i c} \leq \bar{P} x_{i c} \forall i \in \mathcal{S}, c \in \mathcal{C} \\
& x_{i j c} \leq x_{i c} \forall i \in \mathcal{S}, j \in \mathcal{N}, c \in \mathcal{C} \\
& \sum_{i \in \mathcal{S}} h_{i m c} p_{i c} \leq \bar{I}_{m c} \quad \forall m \in \mathcal{M}, c \in \mathcal{C} \\
& g_{i j c}^{i} p_{i c} \geq \bar{\gamma} \sum_{i^{\prime} \in \mathcal{S}, i^{\prime} \neq i} g_{i^{\prime} j c}^{i} p_{i^{\prime} c}+\bar{\gamma} \sum_{i^{\prime \prime} \in \mathcal{S}} g_{i^{\prime \prime} j c}^{i} q_{i^{\prime \prime} c}^{*}+\bar{\gamma} \sum_{k \in \mathcal{T}} l_{k j c}^{i} h_{k c}+
\end{aligned}
$$




$$
\begin{gathered}
\bar{\gamma} N_{c}-\Gamma\left(1-x_{i j c}\right) \quad \forall i \in \mathcal{S}, j \in \mathcal{N}, c \in \mathcal{C} \\
g_{i j c} p_{i c} \geq g_{i^{\prime} j c^{\prime}} p_{i^{\prime} c^{\prime}}-\Gamma\left(1-x_{i j c}\right) \quad \forall i, i^{\prime} \in \mathcal{S}, j \in \mathcal{N}, c, c^{\prime} \in \mathcal{C} \\
x_{i c}, x_{i j c} \in\{0,1\}, p_{i c} \geq 0 \quad \forall i \in \mathcal{S}, j \in \mathcal{N}, c \in \mathcal{C}
\end{gathered}
$$

where $\left\{y_{i j c}^{*}\right\}_{i \in \mathcal{S}, j \in \mathcal{N}, c \in \mathcal{C}}$ and $\left\{q_{i c}^{*}\right\}_{i \in \mathcal{S}, c \in \mathcal{C}}$ are from a set of the optimal solutions to the follower's problem:

$$
\max _{y, Y, q}\left(\sum_{j \in \mathcal{N}} w_{j} \sum_{i \in \mathcal{S}} \sum_{c \in \mathcal{C}} y_{i j c}-\lambda \sum_{i \in \mathcal{S}} \sum_{c \in \mathcal{C}} y_{i c}\right)
$$

subject to

$$
\begin{aligned}
& \sum_{c \in \mathcal{C}}\left(x_{i c}+y_{i c}\right) \leq 1 \quad \forall i \in \mathcal{S} \\
& y_{i j c} \leq y_{i c} \forall i \in \mathcal{S}, j \in \mathcal{N}, c \in \mathcal{C} \\
& q_{i c} \leq \bar{Q} y_{i c} \forall i \in \mathcal{S}, c \in \mathcal{C} \\
& \sum_{i \in \mathcal{S}} h_{i m c} q_{i c} \leq \bar{I}_{m c}-\sum_{i \in \mathcal{S}} h_{i m c} p_{i c} \forall m \in \mathcal{M}, c \in \mathcal{C} \\
& g_{i j c}^{i} q_{i c} \geq \bar{\gamma} \sum_{i^{\prime} \in \mathcal{S}, i^{\prime} \neq i} g_{i^{\prime} j c}^{i} q_{i^{\prime} c}+\bar{\gamma} \sum_{i^{\prime \prime} \in \mathcal{S}} g_{i^{\prime \prime} j c}^{i} p_{i^{\prime \prime} c}+ \\
& \bar{\gamma} \sum_{k \in \mathcal{T}} l_{k j c}^{i} h_{k c}+\bar{\gamma} N_{c}-\Gamma\left(1-y_{i j c}\right) \quad \forall i \in \mathcal{S}, j \in \mathcal{N}, c \in \mathcal{C} \\
& g_{i j c} q_{i c} \geq g_{i^{\prime} j c^{\prime}} q_{i^{\prime} c^{\prime}}-\Gamma\left(1-y_{i j c}\right) \quad \forall i, i^{\prime} \in \mathcal{S}, j \in \mathcal{N}, c, c^{\prime} \in \mathcal{C} \\
& g_{i j c} q_{i c} \geq g_{i^{\prime} j c^{\prime}} p_{i^{\prime} c^{\prime}}-\Gamma\left(1-y_{i j c}\right) \quad \forall i, i^{\prime} \in \mathcal{S}, j \in \mathcal{N}, c, c^{\prime} \in \mathcal{C} \\
& y_{i c}, y_{i j c} \in\{0,1\}, q_{i c} \geq 0 \quad \forall i \in \mathcal{S}, j \in \mathcal{N}, c \in \mathcal{C}
\end{aligned}
$$

Upper level (3)-(12) of the problem corresponds to the leader's problem and controls variables $x, X$, and $p$. Lower level (13)-(21) formalizes the follower's problem (FP) and controls variables $y, Y$, and $q$. The follower, at the lower level, maximizes its profit after the leader's decision, at the upper level. The leader maximizes its profit independently affected by the follower's reaction. We call the entire problem (3)-(21) as the leader's problem (LP) as well because our goal is to find the location of stations provided the maximal leader's profit. Thereby a feasible solution to the LP is defined by the optimal solution to the FP.

The objective functions (3) and (13) can be understood as the total profit obtained respectively by the leader and the follower, computed as the difference between the expected revenue from clients served and the operational costs for 
the stations installed. Constraints (4) state that a client is served either by the leader or the follower. Constraint (5) limits the maximum number of stations that the leader can afford to install. Remember that value $K$ is defined by the leader's initial budget. Constraints (6) and (14) mean that each station can use at most one frequency channel. Constraints (7) and (16) mean that whenever station $i$ is installed its transmit power should be less than threshold value $\bar{P}$ and $\bar{Q}$, respectively. Note that if no station is installed at site $i$, or if station $i$ does not use channel $c$ then the transmit power is necessarily zero because $p_{i c}$ and $q_{i c}$ have been defined as non-negative variables. Constraints (8) and (15) state that a service is possible only if a station is installed. Constraints (9) and (17) are the interference power constraints at each MP on each channel. Constraints (10) are the SINR conditions for a client to be covered. When $x_{i j c}=1$ the expression boils down to the SINR condition about a given SINR threshold. Whenever $x_{i j c}=0$ then the condition is always fulfilled because of the large value of $\Gamma$. In the sums of the Right Hand Side (RHS) of (10), only transmitters with the channel $c$ contribute to the interference in the channel considered. If there is no station at site $i$ (i.e. $p_{i c}=0$ ) then $x_{i j c}$ is necessarily 0 by constraint (8). Note that although the follower has not showed up yet when the leader makes the planning the impact of the interference created by its stations is taken into account in the RHS of (10). Constraints (11) combined with (10) state that the client satisfying the minimal SINR constraint is served by a BS providing the most powerful pilot signal.

Constraints (14) define the set of potential sites for the follower. Constraints (17) state that the interference temperature threshold values must be updated by taking into account the transmissions of the stations installed by the leader. Constraints (18) are the SINR conditions for the follower. Note that the interference created by the leader, appearing in the RHS of (18), is a constant in the FP because the stations of the leader are already installed and configured at the arrival of the follower. Constraints (20) state that the choice between the leader and the follower relies on the most powerful received pilot signal. Notice that, here, the path-loss term assumes omni-directional antennas and not directional antennas. This is due to the fact that the measurement phase should be done equally in all directions.

\subsection{Characterization of the follower's behavior}

Our problem is to find a solution maximizing the leader's profit. Nevertheless if there are several equally optimal solutions to the FP this may result in different leader's objective function values. In other words, it is possible to encounter cases where the leader's profit cannot be calculated unambiguously.

In order to turn the problem into a well posed one we can consider two possible follower's behaviors:

- Cooperative behavior (altruistic follower): in case of multiple optimal solutions to the FP, the follower always selects one of those delivering the maximal profit to the leader. 
- Non-cooperative behavior (selfish follower): in this case, the follower always selects one of the solutions delivering the minimal profit to the leader.

One can easily verify that under such assumptions it is always possible to calculate the leader's profit for any location and configuration of his stations. Note that if the follower's behavior is unknown and cannot thus be predicted considering cooperative and non-cooperative behaviors yields upper and lower bounds to the optimal leader's objective function value, respectively.

\subsection{Analysis of the leader's solution}

Based on the follower's behavior let us characterize the leader's solution. If the follower's solution is unique it means that cooperative and non-cooperative cases coincide. Otherwise, let $F^{*}\left(x^{0}, p^{0}\right)$ be the optimal follower's profit under the leader's solution $x^{0}, p^{0}$. In order to find a cooperative and a noncooperative follower's solution (both are providing the same profit $F^{*}$ ) we have to solve the following auxiliary problems.

Let us first define a set $\mathcal{N}\left(p^{0}\right)$ of the clients which could be served by the leader, i.e. $\mathcal{N}\left(p^{0}\right)=\left\{j \in \mathcal{N} \mid \exists i \in \mathcal{S}: g_{i j c}^{i} p_{i c}^{0} \geq \bar{\gamma}\left(\sum_{i^{\prime} \in \mathcal{S}, i^{\prime} \neq i} g_{i^{\prime} j c}^{i} p_{i^{\prime} c}^{0}+\right.\right.$ $\left.\left.\sum_{k \in \mathcal{T}} h_{k c} l_{k j c}^{i}+N_{c}\right)\right\}$.

In case of cooperative behavior the follower's solution produces the maximal leader's profit and the follower's profit $F^{*}\left(x^{0}, p^{0}\right)$. In other words, we should solve the following Cooperative Auxiliary Problem (CAP) with variables $X, y, Y$, and $q$ :

$$
\text { CAP : } \max _{X, y, Y, q} \sum_{j \in \mathcal{N}\left(p^{0}\right)} w_{j} \sum_{i \in \mathcal{S}} \sum_{c \in \mathcal{C}} x_{i j c}
$$

subject to

$$
\begin{gathered}
\sum_{j \in \mathcal{N}} w_{j} \sum_{i \in \mathcal{S}} \sum_{c \in \mathcal{C}} y_{i j c}-\lambda \sum_{i \in \mathcal{S}} \sum_{c \in \mathcal{C}} y_{i c} \geq F^{*}\left(x^{0}, p^{0}\right) \\
\sum_{i \in \mathcal{S}} \sum_{c \in \mathcal{C}}\left(x_{i j c}+y_{i j c}\right) \leq 1 \quad \forall j \in \mathcal{N} \\
x_{i j c} \leq x_{i c}^{0} \forall i \in \mathcal{S}, j \in \mathcal{N}, c \in \mathcal{C} \\
g_{i j c}^{i} p_{i c}^{0} \geq \bar{\gamma} \sum_{i^{\prime} \in \mathcal{S}, i^{\prime} \neq i} g_{i^{\prime} j c}^{i} p_{i^{\prime} c}^{0}+\bar{\gamma} \sum_{i^{\prime \prime} \in \mathcal{S}} g_{i^{\prime \prime} j c}^{i} q_{i^{\prime \prime} c} \\
+\bar{\gamma} \sum_{k \in \mathcal{T}} h_{k c} l_{k j c}^{i}+\bar{\gamma} N_{c}-\Gamma\left(1-x_{i j c}\right) \forall i \in \mathcal{S}, j \in \mathcal{N}, c \in \mathcal{C} \\
\sum_{c \in \mathcal{C}}\left(x_{i c}^{0}+y_{i c}\right) \leq 1 \quad \forall i \in \mathcal{S} \\
g_{i j c}^{i} q_{i c} \geq \bar{\gamma} \sum_{i^{\prime} \in \mathcal{S}, i^{\prime} \neq i} g_{i^{\prime} j c}^{i} q_{i^{\prime} c}+\bar{\gamma} \sum_{i^{\prime \prime} \in \mathcal{S}} g_{i^{\prime \prime} j c}^{i} p_{i^{\prime \prime} c}^{0}
\end{gathered}
$$




$$
\begin{gathered}
+\bar{\gamma} \sum_{k \in \mathcal{T}} h_{k c} l_{k j c}^{i}+\bar{\gamma} N_{c}-\Gamma\left(1-y_{i j c}\right) \quad \forall i \in \mathcal{S}, j \in \mathcal{N}, c \in \mathcal{C} \\
g_{i j c} q_{i c} \geq g_{i^{\prime} j c^{\prime}} p_{i^{\prime} c^{\prime}}^{0}-\Gamma\left(1-y_{i j c}\right) \quad \forall i, i^{\prime} \in \mathcal{S}, j \in \mathcal{N}, c, c^{\prime} \in \mathcal{C}
\end{gathered}
$$

$(15)-(17),(19),(21)$

$$
x_{i j c} \in\{0,1\} \forall i \in \mathcal{S}, j \in \mathcal{N}, c \in \mathcal{C} .
$$

Let $X^{*}, y^{*}, Y^{*}, q^{*}$ be the optimal solution to this auxiliary problem. Then the leader's profit is given by:

$$
\sum_{j \in \mathcal{N}} w_{j} \sum_{i \in \mathcal{S}} \sum_{c \in \mathcal{C}} x_{i j c}^{*}-\lambda \sum_{i \in \mathcal{S}} \sum_{c \in \mathcal{C}} x_{i c}^{0}
$$

In case of non-cooperative behavior let us introduce new auxiliary variables: $z_{i j c} \in\{0,1\}, i \in \mathcal{S}, j \in \mathcal{N}\left(p^{0}\right), c \in \mathcal{C} . z_{i j c}$ is 1 if client $j$ is served neither by the leader nor by the follower because of a low SINR. The Non-Cooperative Auxiliary Problem (NCAP) is obtained by replacing the objective function (22) by:

$$
\operatorname{NCAP}: \max _{X, y, Y, q, z} \sum_{j \in \mathcal{N}\left(p^{0}\right)} w_{j} \sum_{i \in \mathcal{S}} \sum_{c \in \mathcal{C}}\left(y_{i j c}+z_{i j c}\right) \text {, }
$$

which means that the follower intends to catch as many clients as possible, and by adding to (23)-(30), (15)-(17), (19), (21) the following constraints:

$$
\begin{array}{r}
\sum_{i \in \mathcal{S}} \sum_{c \in \mathcal{C}}\left(x_{i j c}+y_{i j c}+z_{i j c}\right)=1 \quad \forall j \in \mathcal{N}\left(p^{0}\right) \\
g_{i j c}^{i} p_{i c}^{0}<\bar{\gamma} \sum_{i^{\prime} \in \mathcal{S}, i^{\prime} \neq i} p_{i^{\prime} c}^{0} g_{i^{\prime} j c}^{i}+\bar{\gamma} \sum_{i^{\prime \prime} \in \mathcal{S}} g_{i^{\prime \prime} j c}^{i} q_{i^{\prime \prime} c}+ \\
\bar{\gamma} \sum_{k \in \mathcal{T}} h_{k c} l_{k j c}^{i}+\bar{\gamma} N_{c}+\Gamma\left(1-z_{i j c}\right) \quad \forall i \in \mathcal{S}, j \in \mathcal{N}\left(p^{0}\right), c \in \mathcal{C} .
\end{array}
$$

Constraints (33) mean that each client is served by the leader, or is caught by the follower, or is not served at all. Constraints (34) guarantee that the client $j$ is not served from station $i$ on the channel $c$, i.e. $z_{i j c}=1$, if the SINR is too low. 


\section{Complexity analysis}

In this section we study the computational complexity of LP (3)-(21) and FP (13)-(21). We will show that the both of the above optimization problems are hard to solve. In fact, we claim that the first problem is $\Sigma_{2}^{P}$-hard for cooperative and non-cooperative follower's behavior and the follower problem NP-hard in the strong sense. The class $\Sigma_{2}^{P}$ is a part of the polynomial time hierarchy. It contains all decision problems which can be described by the formula of the form $\exists x_{1}, \ldots, \exists x_{l} \forall y_{1}, \ldots, \forall y_{k} \varphi(x, y)$ where $\varphi(x, y)$ is a quantifier free formula. It is widely assumed that the class $\Sigma_{2}^{P}$ is a proper superset of the class NP. Thus, the leader problem turns out to be even more difficult than NP-complete problems.

The hardness proof uses a reduction from $\exists x_{1}, \ldots, \exists x_{l} \forall y_{1}, \ldots, \forall y_{k}$ SAT decision problem. We recall now some preliminaries. A set $\mathcal{X}$ denotes the set of logical variables. If $x \in \mathcal{X}$ is a variable, than $\neg x$ is its negation. The set $\{x, \neg x \mid x \in \mathcal{X}\}$ is the set of literals. A term is a conjunction of literals. A formula in 3 -DNF is a disjunction of terms where each term contains exactly three literals. The decision problem $\exists x_{1}, \ldots, \exists x_{l} \forall y_{1}, \ldots, \forall y_{k}$ 3SAT is defined as follows. Given a formula $\varphi(x, y)$ in 3 -DNF over a partitioned set $\mathcal{X} \cup \mathcal{Y}$ of variables, decide whether the formula $\exists x_{1}, \ldots, \exists x_{l} \forall y_{1}, \ldots, \forall y_{k}$ 3SAT is satisfied, where $x_{i} \in \mathcal{X}$ and $y_{j} \in \mathcal{Y}$. It is well known that this decision problem is $\Sigma_{2}^{P}$-complete [29]. But we need a modified variant of this statement. It is easy to verify that the decision problem remains $\Sigma_{2}^{P}$-complete even each term contains exactly one $x$ variable and two or three $y$ variables. This special case of the decision problem is denoted as $\exists x_{1}, \ldots, \exists x_{l} \forall y_{1}, \ldots, \forall y_{k} 3,4 \mathrm{SAT}$.

Lemma 1 [9] The problem $\exists x_{1}, \ldots, \exists x_{l} \forall y_{1}, \ldots, \forall y_{k} 3,4 S A T$ is $\Sigma_{2}^{P}$-complete.

This decision problem was used to study the complexity of the $(r \mid p)$ centroid problems. Below we adopt the same ideas to the leader problem. To that end, we introduce three types of clients: rich, regular, and poor. The rich clients patronize the leader stations. The regular clients patronize the follower stations. The poor clients create an area of competition. The formula $\exists x_{1}, \ldots, \exists x_{l} \forall y_{1}, \ldots, \forall y_{k} 3,4$ SAT will satisfied if and only if at least one poor client patronizes the leader station in the optimal solution to the leader problem (at least one term is True).

Theorem 1 The LP is $\Sigma_{2}^{P}$-hard for cooperative and non-cooperative follower's behavior.

Proof We reduce $\exists x_{1}, \ldots, \exists x_{l} \forall y_{1}, \ldots, \forall y_{k} 3,4$ SAT to the LP. For each instance of the decision problem, we create an instance of the LP. Set $|\mathcal{C}|=|\mathcal{S}|,|\mathcal{M}|=$ $|\mathcal{T}|=0$. In other words, for each site $i \in \mathcal{S}$, we can assign a channel $c(i) \in$ $\mathcal{C}$ and replace the variables $x_{i c}$ by $x_{i c(i)}$ and replace variables $y_{i c}$ by $y_{i c(i)}$. Moreover, constraints (9) and (17) vanish. We put $g_{i j c}^{i}=1 / d_{i j}$, where $d_{i j}$ is Euclidean distance between client $j$ and station $i$ and $\bar{P}>\bar{Q}$. In this case, each leader's station can serve all the clients in a disk of radius $R_{L}=R_{L}\left(\bar{P}, \bar{\gamma}, N_{c}\right)$. 


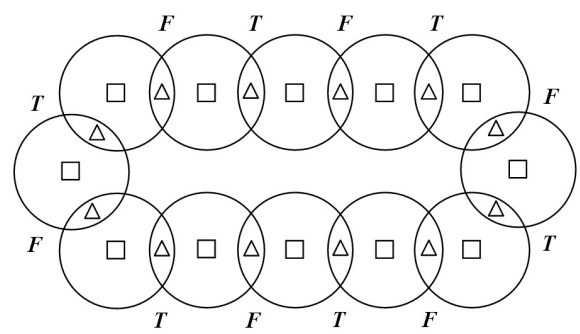

Fig. 2 A circuit for Boolean variable $x_{i}$ or $y_{j}$ in the reduction. Squares indicate clients, triangles are sites for stations, $\mathrm{T}=$ True, $\mathrm{F}=$ False

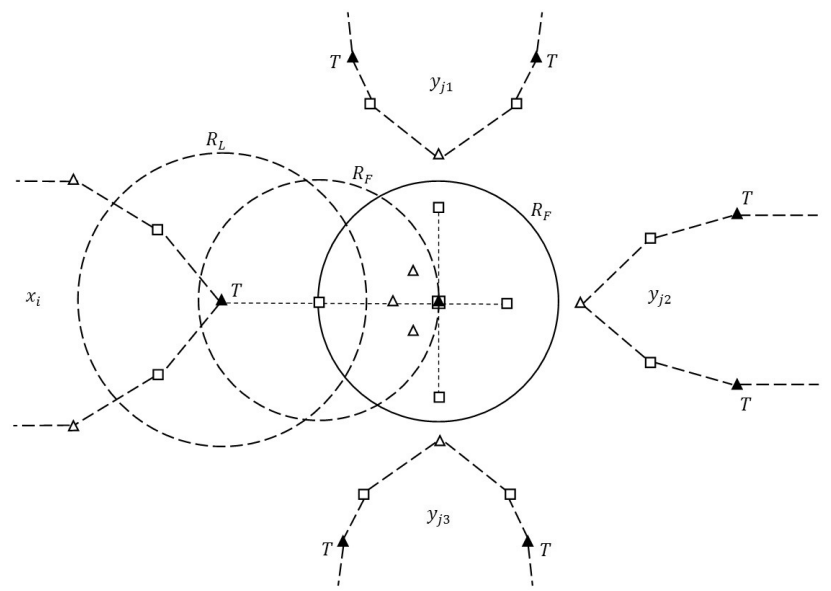

Fig. 3 Configuration of circuits for term $\left(x_{i} \wedge y_{j_{1}} \wedge y_{j_{2}} \wedge y_{j_{3}}\right)$
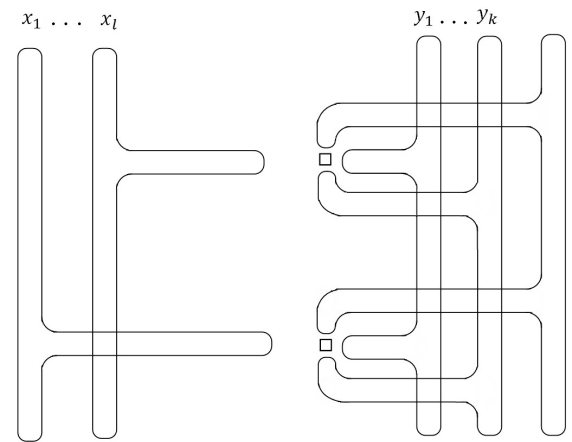

Fig. 4 A schematic plan of the reduction to the LP

Each follower's station can serve all the clients in a disk of radius $R_{F}=$ $R_{F}\left(\bar{Q}, \bar{\gamma}, N_{c}\right)$. Without loss of generality we assume that $R_{L}=1$ and $R_{F}=$ $1-\delta$ for some positive $\delta$. According to (11),(19),(20) each client patronizes the nearest station of the leader or the follower. In case of ties, the followers station is preferred. 


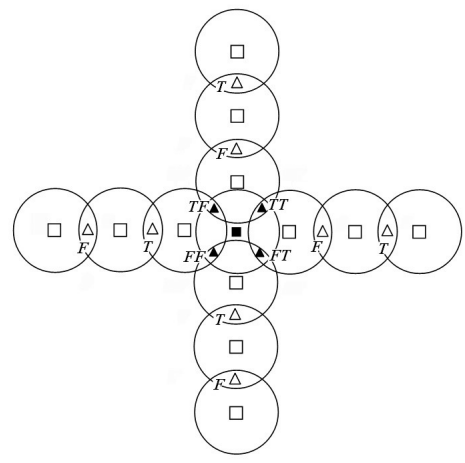

Fig. 5 A configuration of the circuits for a junction

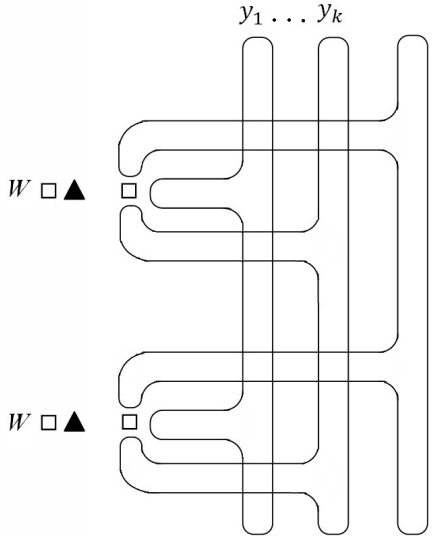

Fig. 6 A schematic plan of the reduction to the FP

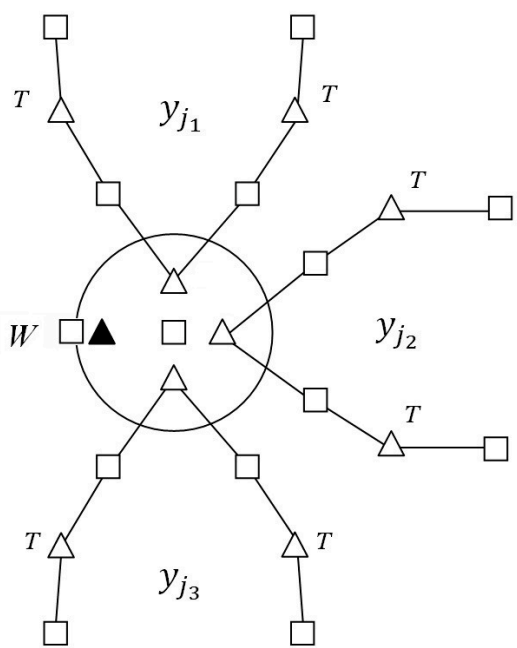

Fig. 7 A clause configuration 
For each variable $x_{i}$ or $y_{j}$ of the decision problem, we create the circuit of circles (see Fig. 2). The radius of each circle is 1 for variable $x_{i}$ and $1-\delta$ for variable $y_{j}$. We locate a client in the center of each circle. The client has a positive weight $w_{x}$ (rich client) or $w_{y}$ (regular client) depending on the type of variable. We assume that $w_{y}<w_{x}$. The distance between centers of the neighboring circles for variable $x_{i}$ is $(2-\varepsilon)$ for some positive $\varepsilon, \varepsilon<\delta$. Similar, for variable $y_{j}$, this distance is $(2-2 \delta-\varepsilon)$.

The number of circles in each circuit is even, say $2 \tau$. If the circuit corresponds to variable $x_{i}$, and $K=\tau, \lambda<2 w_{y}<2 w_{x}$ then the leader's optimal solution is to open $K$ stations in the intersections of the circles and serve all clients. Note that the follower cannot serve these clients because $R_{F}<1-\varepsilon / 2$. The leader can do that in two different ways. One of them will correspond to True value of $x_{i}$, another one - False value (see Fig. 2). A similar idea is used to prove the complexity results for the Euclidean $p$-center problem [24] and for the $(r \mid p)$-centroid problems [9].

For each term we introduce five additional (poor) clients which are located in a circle with radius $R_{F}$ (see Fig. 3). Four of them with a positive weight $w, w<w_{y}$ are inside of the circle. The last client with weight $w / 2$ is on the boundary of the circle. We assume that $\lambda<3.5 \mathrm{w}$. Hence, the follower will capture these five clients if he installs own station at the center of the circle. But the leader will try to capture the last client as well. To block such intervention, we introduce three additional sites near the center of the circle as presented in Fig. 3. Each of them allows the follower to capture four clients only including the client with weight $w / 2$ on the boundary of the circle. As a result, all clients will be served, but the last client with weight $w / 2$ is the subject for competition. Let us consider the term $\left(x_{i} \wedge y_{j_{1}} \wedge y_{j_{2}} \wedge y_{j_{3}}\right)$. The circuits for these variables are located near the circle as Fig. 3 shows. If the True assignment corresponds to black triangles in the circuits, then the follower installs own station in the center of circle and captures four clients with total weight $4 \mathrm{w}$. The client on the boundary of the circle patronizes the leader station in the $x$-circuit. For other assignments, this client patronizes the follower station at the center of circle or in a nearest site. In other words, the only one assignment (indicated in Fig. 3) corresponds to the case when the client on the boundary of the circle patronizes the leader's station. In this case the term is True. If the leader captures at least one such client, the formula is satisfied. A schematic plan of allocation of these term configurations and their relationship with the circuits is shown in Fig. 4.

As we can see, the circuits have mutual intersections or junctions. But the junctions correspond to pairs $x_{i} x_{j}$ or $y_{i} y_{j}$ only. We have no junction for pairs $x_{i} y_{j}$. Let us consider such junction in details and present a configuration to save the parity. To this end, we introduce an additional client with the weight $w_{x}$ or $w_{y}$ and put it in the center of the junction as it is shown by black square in Fig. 5. Moreover, we add four sites indicated by black triangles in Fig. 5. The decision maker can capture three clients by installing station in one of these sites. Following [24], we require that the number of clients between two nearest junctions for the same circuit will be odd. 
We claim that the optimal solution for the instance of the LP indicates whether the formula $\exists \forall 3,4 S a t$ is satisfied or not. Let $n_{t}$ be the number of terms, $p_{x}$ be the number of circles for $x$ circuits, $q_{x}$ be the number of their junctions, $p_{y}$ and $q_{y}$ be the number of circles and junctions for $y$ circuits. We set $K=0.5 p_{x}, \lambda=0.5\left(w_{y}+w_{x}\right)$. In this case, the leader captures $p_{x}$ clients in the center of circles for $x$ circuits and $q_{x}$ clients for junctions because $w_{x}>w_{y}$. The remaining $5 n_{t}+p_{y}+q_{y}$ clients are distributed between the leader and the follower. But $4 n_{t}+p_{y}+q_{y}$ clients will be captured by the follower because $\lambda<2 w_{y}$ and $\lambda<3.5 w$. The $n_{t}$ clients for the terms will patronize the leader or the follower stations depending on the parity of the solution. But all clients will be served. The leader installs $K$ stations, the follower installs $0.5 p_{y}+n_{t}$ stations. Hence, the sum of the objective functions (3), (13) is a constant $\sum_{j \in \mathcal{N}} w_{j}-\lambda\left(K+0.5 p_{y}+n_{t}\right)$. In this case the cooperative and noncooperative cases coincide. Now it is easy to see that the formula is satisfied if and only if the leader will get at least one poor client with weight $w / 2$.

To complete the proof, we should note that our reduction is polynomial.

Theorem 2 The FP is NP-hard in the strong sense.

Proof Let us consider the well-known 3Sat problem which is NP-hard in the strong sense. We are given a Boolean formula in the conjunctive normal form. Each clause includes exactly three literals. We need to decide whether this formula is satisfied.

We reduce this decision problem to the FP. To that end, we just modify the previous reduction in Fig. 3 and Fig. 4. Again, for each Boolean variable $y_{j}$, we create a circuit, which consists of even number of circles with radius $R_{F}$. For each clause we introduce two clients and a site for station. One rich client has the weight $W, W>\lambda$. Another poor client has the positive weight $w$, such that $\lambda>w$ (see Fig. 6).

The distance between these two clients is $R_{F}$. New site for the station is located between the clients. The poor client is located at the center of circle. Fig. 7 shows a configuration of circuits for clause $\left(y_{j_{1}} \vee y_{j_{2}} \vee y_{j_{3}}\right)$. If at least one variable is True, then the client is served by the follower. Otherwise, the client is served by the leader as it is shown in Fig. 7 .

Let $n_{c l}$ be the number of clauses. We set $K=n_{c l}$. The leader opens $K$ stations capturing all clients with weight $W$. Later on, the follower opens $0.5 p_{y}$ stations at the intersections of circles and gets at least $\left(p_{y}+q_{y}\right)$ clients with the weight $w_{y}$. The remaining $n_{c l}$ clients with the weight $w$ are distributed between the leader and the follower. The formula is satisfied if and only if the follower captures all these clients. Hence, the follower problem is NP-hard. Now we wish to show that it is NP-hard in the strong sense.

Let us return to the schematic plan of the reduction (see Fig. 6). We can see that the plan is decomposed on $n_{c l}$ separated regions, one for each clause. In each region, we have the circles for $y$ circuits. The number of these circles is linear in $k$. But we need exact coordinates $\left(z_{1}, z_{2}\right)$ for each client. It is easy to see that there are two constants $c_{1}$ and $c_{2}$ such that $z_{1} \leq c_{1} k$ and $z_{2} \leq c_{2} n_{c l}$ for all clients. Without loss of generality we can assume that centers of all 
circles have coordinates with polynomial encoding length. Otherwise, we can slightly move the centers of circles by vary $\delta$. Similar arguments are valid for the stations and rich clients. Hence, if we put $W=3, w_{y}=2, w=1$, then we get the desired.

Despite these complexity results, we present in Section 6 an efficient matheuristic for the LP which allows us to find near optimal solutions for the cooperative and non-cooperative follower's behaviors.

\section{Matheuristic for the bi-level problem}

Sometimes matheuristics called "model-based metaheuristics" [7], are heuristic algorithms made by the interoperation of metaheuristics and mathematical programming techniques. In this section, we describe a matheuristical approach based on a probabilistic tabu search (TS) and mathematical programming that can be used by the leader to choose position, operational channel and transmission power for its stations. A similar approach has been successfully applied for bi-level problem, namely, the discrete $(r \mid p)$-centroid problem in [3]. The fundamental ideas of TS have been proposed by Glover for single level problems. We refer the reader to [13] for an introduction to TS.

The matheuristic that we propose to solve the LP is formalized in Algorithm 1. Let us discuss it in details. Noticing that some leader's variables namely, $x$ are binary, our local search procedure proceeds in the space of these variables. We initially create a good starting solution to the LP by ignoring the arrival of the follower, i.e. we solve the LP with $q^{*}=Y^{*}=y^{*}=0$. The leader variables $\left\{p_{i c}\right\}$ are assigned to their maximal transmit power under constraints (7) and (9). This step provides the initial values of variables $\left\{x_{i c}\right\}$.

Then we run $t_{\max }$ iterations of TS. At each iteration we first generate a randomized neighboring by exploring the Swap-Flip neighborhood of $x$. It means that a neighbor solution is obtained from the current solution by installing and/or uninstalling a station, or by changing the channel used by a leader's station. As exploring this entire neighborhood of size $O(k(|\mathcal{S}|-k)|\mathcal{C}|)$ might be time-consuming we use a randomization procedure, which independently includes in the randomized neighborhood (denoted $N_{p r}$ ) each element of the Swap-Flip neighborhood with a fixed probability $\mathrm{pr}$.

For each element $x^{\prime} \in N_{p r}(x)$ we know the leader's stations and channels (i.e., the leader's variables $x$ are fixed). In order to obtain leader's transmit power $p\left(x^{\prime}\right)=\left\{p_{i c}\right\}$ for the leader's stations we solve the power allocation problem by means of CPLEX (line 6). Specifically, that means that we solve the LP where the leader's variables $\left\{x_{i c}\right\}$ are fixed and $q_{i c}^{*}=y_{i j c}^{*}=y_{i c}^{*}=0$.

Then to find the values of leader's variable $X$ and calculate the leader's profit we need the optimal solution to FP (13)-(21). This problem is NPhard and is solved by mathematical programming tools (here a branch-and-cut algorithm from CPLEX 12.3.0).

If the solution to the FP is unique, we take the optimal solution vectors $\left\{y_{i j c}^{*}\right\},\left\{q_{i c}^{*}\right\}$ and inject them into the LP. Then we derive $\left\{x_{i j c}\right\}$, whose ele- 
ments are used in (3) to calculate the leader's profit at the current iteration (line 11). If there are multiple solutions to the FP we solve CAP (if a cooperative follower's behavior is assumed) or NCAP (non-cooperative behavior). Recall that if the follower's behavior is not known, CAP provides an upper bound to the leader's profit while NCAP yields a lower bound. Using the optimal follower's solution we calculate $X$ and the leader's profit (line 13) using (31).

In order to reduce memory requirements the Tabu list contains only some components of the leader's solutions, namely the pairs or triplets of the installed/uninstalled stations and the channel, which has been changed during the move to the best neighbor solution. The length of Tabu list is a constant denoted as TT (for Tabu Tenure).

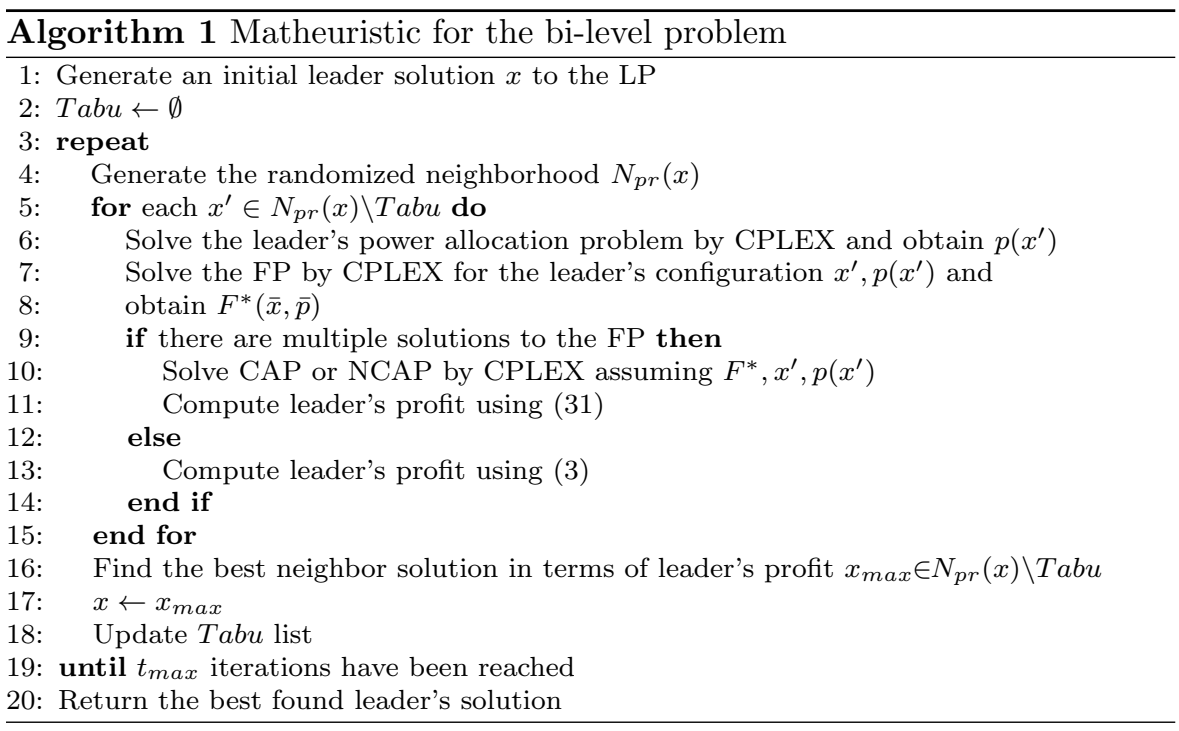

\section{Discussion}

In this section, we discuss the consistency of our generic model with respect to the IEEE 802.22 standard, according to the recent suggestions/ruling from the US FCC and the UK Ofcom. In addition, we trace research directions that we plan to follow in order to make our work more complete and realistic.

- Static nature of the stations: In this paper, we assume that DTV channel allocation and stations operational channels will not be modified in future. The study of the case where some channels are dismissed/added by the PU is left for future work.

- Spectrum database model: In the context of IEEE 802.22, regulators are moving away from the interference power constraint approach to the 
spectrum database model. In this model, providers obtain from a central database the set of available channels and the maximum transmit power on available channels for every location. Our model is more generic in the sense that it does not require a central entity but only a communication between the provider and the local PU transmitters about the location of the MPs and the interference constraints. However, we can easily introduce the database model in our equations by removing (9) and (17) and by replacing $\bar{P}$ and $\bar{Q}$ with $\left\{\bar{P}_{i c}\right\}$ and $\left\{\bar{Q}_{i c}\right\}$ in (7) and (16) respectively. $\bar{P}_{i c}$ and $\bar{Q}_{i c}$ are the maximum allowable transmit powers on location $i$ on channel $c$ and can be obtained from the database. $\left\{\bar{P}_{i c}\right\}$ and $\left\{\bar{Q}_{i c}\right\}$ are set to 0 if $c$ is not allowed. Note that $\left\{\bar{P}_{i c}\right\}$ and $\left\{\bar{Q}_{i c}\right\}$ are constant in our model because of the assumptions that 1) the PUs have static nature and 2) the leader exploits channels which are allowed for fixed devices only ${ }^{2}$, meaning that portable devices cannot perform transmissions and therefore interfere with the signals emitted by the stations.

- Simultaneous arrival of providers: The present work is motivated by the dynamics of low competitive markets which are typical of rural areas, where providers can hesitate to invest because of the high uncertainty on future profit margins. In this context, our objective is to furnish a support to decision making for the first provider getting to market. However, if the market taken into consideration is either high competitive and/or characterized by fast competitive reactions, then the case where two or more providers get to market at the same time is definitely possible. This new scenario requires a separate analysis which is left for future work.

- Uplink communications: In IEEE 802.22 the clients are possibly equipped with directional antennas for uplink transmissions. The emitted signals create interference to the MPs, as well as to other stations and clients which are receiving data on the same channel. We can easily integrate the uplink communications in our model by adding a new SINR constraint on the uplink for a client to be covered and by considering the interference created by clients on MPs.

- Uncertainty on clients positions: In our model, the provider is fully aware of the client locations and demands. A possible further work is to assume a certain uncertainty on the client positions and a certain probability of client appearance or demise.

- Channel bonding and aggregation: In the case of more demanding clients (in terms of connection speed), one can think of making use of channel bonding/aggregation ${ }^{3}$. In order to integrate such feature in our model, it is enough to allow stations and clients for communications over multiple channels. To this end, define new variables $x_{i} \in\{0,1\}$ and $y_{i} \in$

2 In the United States, for instance, we are talking about the VHF channels 2, 5, 6, 7 $13,14-20$ and the UHF channels $14-20$.

3 When two or more channels in the frequency domain are available, if these channels are contiguous with each other, they could be bonded as one client channel. Otherwise they could be aggregated meaning that multiple channels at different frequencies are assembled as a common channel. 
$\{0,1\}$ which are one when a station is installed on site $i$ by the leader and the follower, respectively. Then, remove (6), replace $x_{i j c}$ and $y_{i j c}^{*}$ in (4) with $x_{i}$ and $y_{i}^{*}$ and $x_{i c}$ and $y_{i c}$ in (14) with $x_{i}$ and $y_{i}$. In the case of channel bonding, we also need to index the channels and to write a linear constraint that prevents the stations from bonding non-adjacent spectrum slices.

- Stations reconfigurability: In our model, we assume that the stations cannot change the operational channel, once the latter has been chosen. Relaxing such a constraint implies that the leader has to solve a multi-step Stackelberg game at each iteration of the matheuristic algorithm, meaning that the leader can rearrange the operational channel of its installed stations depending on the follower's station configuration. Similarly, the follower can also modify the design of its stations after observing a new leader's strategy. The study of such game is left for the further work.

- Co-siting: In the model presented we do not allow for co-siting, i.e., we do not allow the follower to use the same leader's site. This may be not realistic in many markets where telecommunications regulatory bodies may impose the leader to host competitors' antennas (on payment) on the installed towers. Such situation can be supported by our model by modifying the FP in the following manner: define set $\mathcal{S}^{L}$ containing the sites where the leader has installed its stations and set $\mathcal{S}^{F}:=\mathcal{S} \backslash \mathcal{S}^{L}$. Remove constraints (14) and rewrite the objective follower's function as follows:

$$
\max _{y, Y, q}\left(\sum_{j \in \mathcal{N}} w_{j} \sum_{i \in \mathcal{S}} \sum_{c \in \mathcal{C}} y_{i j c}-\lambda \sum_{i \in \mathcal{S}^{F}} \sum_{c \in \mathcal{C}} y_{i c}-\lambda^{\prime} \sum_{i \in \mathcal{S}^{L}} \sum_{c \in \mathcal{C}} y_{i c}\right)
$$

where $\lambda^{\prime}$ is the cost per time unit paid by the follower for its transmission equipment on the leader's tower.

- Failure to follower arrival: the probability of the follower arrival as a function of time should be carefully estimated by the leader before deploying the stations at a secondary network. A solution which is more robust to the arrival of a follower might in fact produce less profits (and therefore a loss) if compared to the optimal solution in monopoly regime. The evaluation of such loss is left for the future work.

\section{Numerical work}

In this section we conduct simulations to evaluate the performance of the proposed matheuristics, the adequacy of the mathematical model on realistic instances and demonstrate the benefit brought by the competitive approach over the non-competitive one.

\subsection{Simulation parameters and scenario considered}

We list in Table 1 all the parameter values and place aside its value range in real world applications $[8,22,23,28,31]$. We consider 50 potential station sites 
and 40 clients distributed on a square rural area of side $150 \mathrm{Km}$. The clients are equipped with directional reception antennas characterized by a maximal gain in boresight direction of $16 \mathrm{dBi}$, a $3 \mathrm{~dB}$ beamwidth of $70^{\circ}$ and a front-to-back power ratio of $25 \mathrm{~dB}$. The gain of the antenna is then calculated according to equation (3) in [23].

We set the $w_{j}$ equal to 5 for the client $j$ with cartesian coordinates $(112,118)$ (of the square area defined above) and one for all the other clients. This can represent a situation, where the client with higher priority is a company, which, once served, is expected to pay 5 times more than a ordinary client.

We consider 2 PU DTV towers of height $200 \mathrm{~m}$ operating on 7 channels in the frequency range 470-512 MHz (each TV channel uses a $6 \mathrm{MHz}$ bandwidth). The first PU DTV tower has cartesian coordinates $(0,0)$ (bottom-left corner of the square area defined above) and operates on channels 14 through 17. The second PU DTV tower has coordinates $(150,150)$ (top right corner) and operates on channels 15-18-19-20 (channel 15 is common to the two towers). Note that white spaces found in the VHF range $54-216 \mathrm{MHz}$ and in the UHF range $470-512 \mathrm{MHz}$ are exploitable by all unlicensed devices but portable [12] (here including wireless microphones). They are therefore particularly suitable for long-term cognitive network planning.

According to FCC regulations, DTV service is defined to exist where the strength of the received useful signal exceeds a certain threshold value which is dependent on the channel frequency. For channels 14 through 69, corresponding to the frequency range $470-806 \mathrm{MHz}$, such value has been fixed to $41 \mathrm{dBu}$. We fix the PU DTV tower transmit power to $80 \mathrm{dBm}$ EIRP, so that we can calculate a protection $F(50,90)^{4}$ contour of approximately $70 \mathrm{Km}$. Along such contours the PU distributes two sets of 4 MPs (8 in total). At each MP the interference temperature created by the station must be kept under a threshold value of $-106 \mathrm{dBm}$. Doing so the PU makes sure that the cognitive network will not disrupt any DTV receivers of the service. Path-losses from PU DTV towers and from station are computed with the $F(50,90)$ propagation curves and with the Okumura-Hata model for rural areas, respectively.

For the simulations, we use MATLAB to implement our algorithm, GAMS for mathematical optimization programming and CPLEX as an optimization solver. The computer is equipped with $4 \mathrm{~GB}$ of RAM and an Intel(R) Core(TM) Duo CPU clocked at $2.8 \mathrm{GHz}$. In the figures, the allocated power is linearly proportional to the size of the circle around each installed station. Working channels are then specified in proximity of each circle and for easy reading a color has been assigned to each DTV channel (red corresponds to channel 17, for instance).

We also assume $K=5, \lambda=0.5, \bar{\gamma}=10 \mathrm{~dB}$, a client height of $9 \mathrm{~m}$, a station feeder loss of $1 \mathrm{~dB}$, a station height of $30 \mathrm{~m}$, a PU DTV tower of $200 \mathrm{~m}$, a PU DTV tower power of $80 \mathrm{dBm}$, a temperature threshold of $-106 \mathrm{dBm}$. For TS, we take $p r=0.18, t_{\max }=10^{4}$ and $T T=30$.

\footnotetext{
$4 F(X, Y)$ represents the spatial and temporal relationship of the TV signal propagation as specified in [15]. It represents the field strength that would exceed a certain threshold at $\mathrm{X} \%$ of locations for $\mathrm{Y} \%$ of time.
} 
Table 1 Simulation Parameters

\begin{tabular}{|l|c|c|}
\hline Parameter & In simulation & Value range \\
\hline Antenna maximum gain in boresight direction & $16 \mathrm{~dB}$ & $11-40 \mathrm{dBi}$ \\
\hline 3dB beamwidth & $70^{\circ}$ & $35^{\circ}-75^{\circ}$ \\
\hline Antenna front-to-back power ratio & $25 \mathrm{~dB}$ & $14-50 \mathrm{~dB}$ \\
\hline DTV tower height & $200 \mathrm{~m}$ & $30-1200 \mathrm{~m}$ \\
\hline DTV tower transmit power & $80 \mathrm{dBm} \mathrm{EIRP}$ & $36-96 \mathrm{dBm} \mathrm{EIRP}$ \\
\hline Interference power threshold & $-106 \mathrm{dBm}$ & $-130,-95 \mathrm{dBm}$ \\
\hline CR-BS feeder loss & $1 \mathrm{~dB}$ & $0.1-3 \mathrm{~dB}$ \\
\hline CR-BS height & 30 & $10-50 \mathrm{~m}$ \\
\hline Number of available channels & 7 & $0-7$ \\
\hline Minimum SINR & 10 & $10-15$ \\
\hline
\end{tabular}
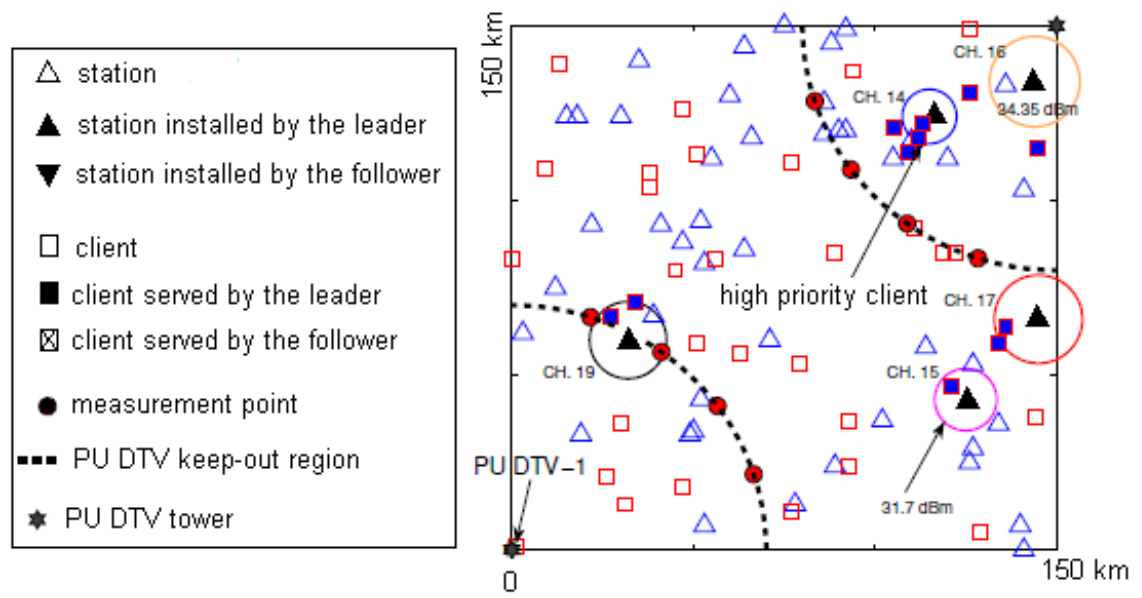

Fig. 8 Best found solution to the LP without taking into account the follower's existence (i.e., $q^{*}, Y^{*}, y^{*}$ taken as zero)

\subsection{Simulation results}

In Fig. 8 we show the best found non-competitive solution to the LP. With this solution, the leader is able to make a profit of 12.5: it installs 5 stations (it could not be installed more stations due to the budget constraints), which cover 14 standard stations plus the high priority client.

In Fig. 9 we show what happens when a second operator with cooperative behavior gets to a market and finds the shortsighted leader's stations configuration displayed in Fig. 8. The follower captures most of clients (among which the high priority client) so that the leader's profit gets as low as 1.5, with a decrease in profits of $88 \%$. It is therefore evident how a configuration, which was optimal in monopoly regime, turns out to be disastrous in the face of 
competition. Notice also that following follower's arrival, two clients that were served by the leader are now unserved due to the interference created by the follower's stations on DTV channel 19.

In Fig. 10 the best found competitive leader's solution by means of Algorithm 1 is depicted. A cooperative-behaved follower has been assumed so that CAP has been solved at each iteration of our algorithm. We find out that the optimal leader's profit is equal to 10.5, that is 6 times more with respect to the case shown in Fig. 9.

We now want to show how the knowledge of the follower's behavior plays an important role in the strategic placement of the leader's stations. With this in mind, we take the optimal leader's stations configuration for cooperative follower (Fig. 9) and see what happens if we turn the follower into a selfish one, willing at the same time to maximize its profits and minimize the profit of the competitor. Thus, we solve the NCAP by setting $F^{*}$ equal to 5.5 , which is the optimal solution to the FP (this can be also verified by looking at Fig. 10). The result of this operation is depicted in Fig. 11. One can easily notice that although the follower's profit is still 5.5, the leader's situation has significantly changed with respect to the one displayed in Fig. 10. Due to the disruptive interference created by the follower, the leader looses two standard clients and it is forced to uninstall a station which had become failing, as it was not covering any client. Its new profit is as much as 9, which is the lower bound of the future leader's profits in a competitive market and which is still higher than the profit obtained without taking into account the arrival of the follower. The upper bound is 10.5, which corresponds to the assumption of a cooperative follower.

In Fig. 12-17 we present simulation results for different scenarios. In Fig. 1214 we show the case where the DTV towers operate on a different number of channels so that the clients can choose among only 3 channels. PU DTV-1 operates on channel 1; PU DTV-2 operates on channels 2 and 3. In Fig. 15-17 we show the case with 3 DTV towers.

In Fig. 18 the behavior of the applied matheuristic is presented. We show how the leader's profit is changed at each iteration of the algorithm under the cooperative follower's behavior for the scenario with 3 PU DTV towers. The best found solution was obtained after 5000 iterations out of 10000 iterations done.

In Fig. 19, 20 execution times of proposed matheuristic are reported with respect to the number of clients and station sites under the cooperative follower's behavior.

\section{Conclusion}

We have considered the problem of placing CR base stations, while anticipating the arrival of a competitor. We find this problem as an interesting application of competitive facility location problems in telecommunication area. We have formalized this problem in terms of bi-level programming and use linear bi- 


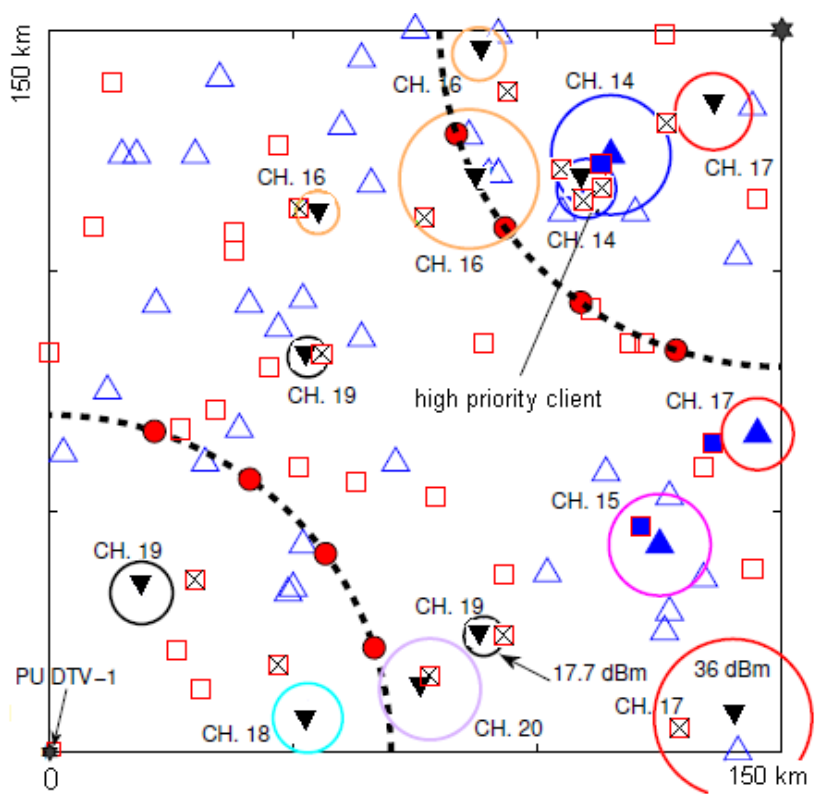

Fig. 9 Optimal reply of the cooperative-behaved follower faced with the initial leader's solution

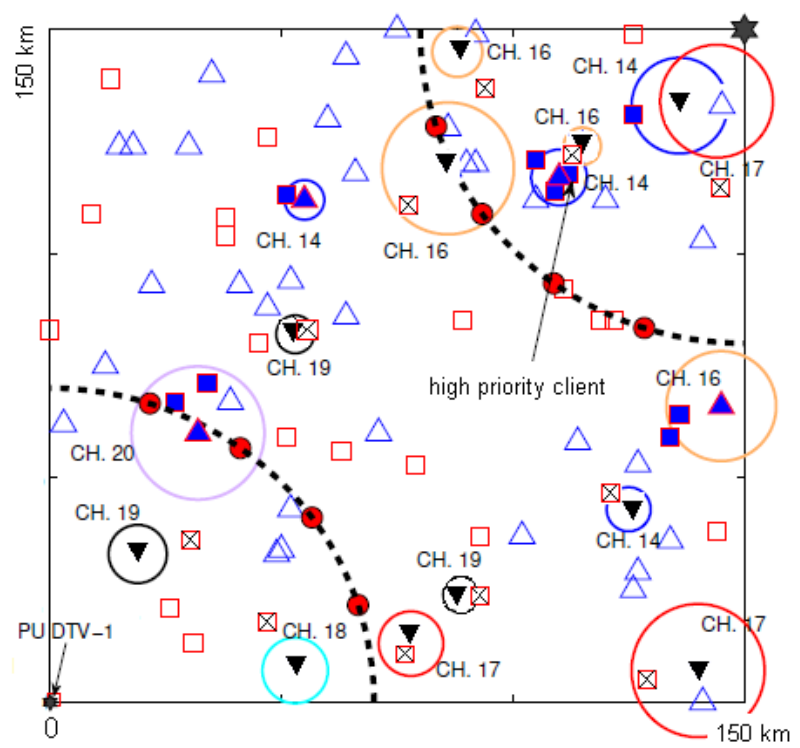

Fig. 10 Best found solution to the LP with cooperative-behaved follower 


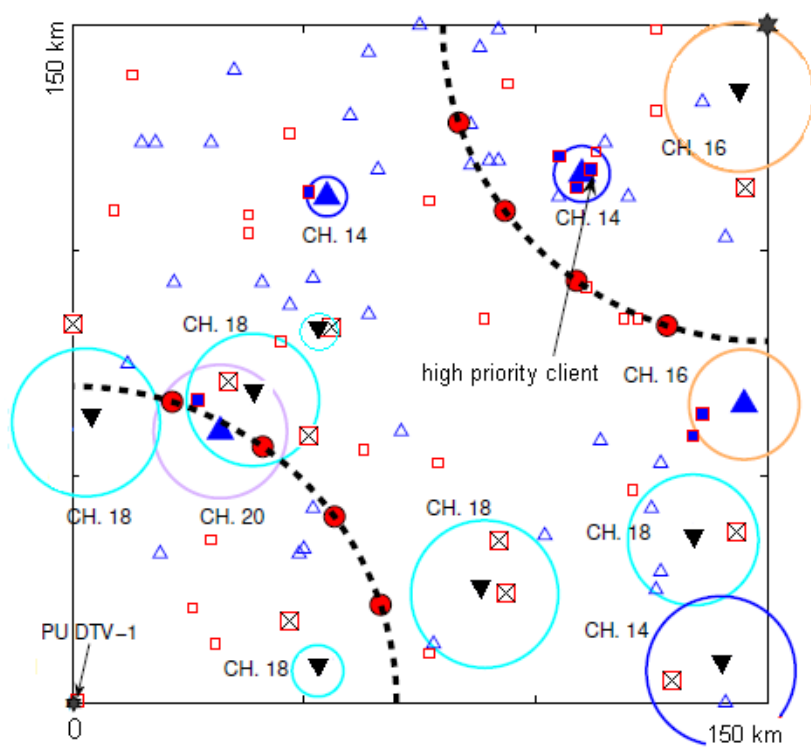

Fig. 11 Non-cooperative follower's solution faced with the leader's stations configuration shown in Fig 10.

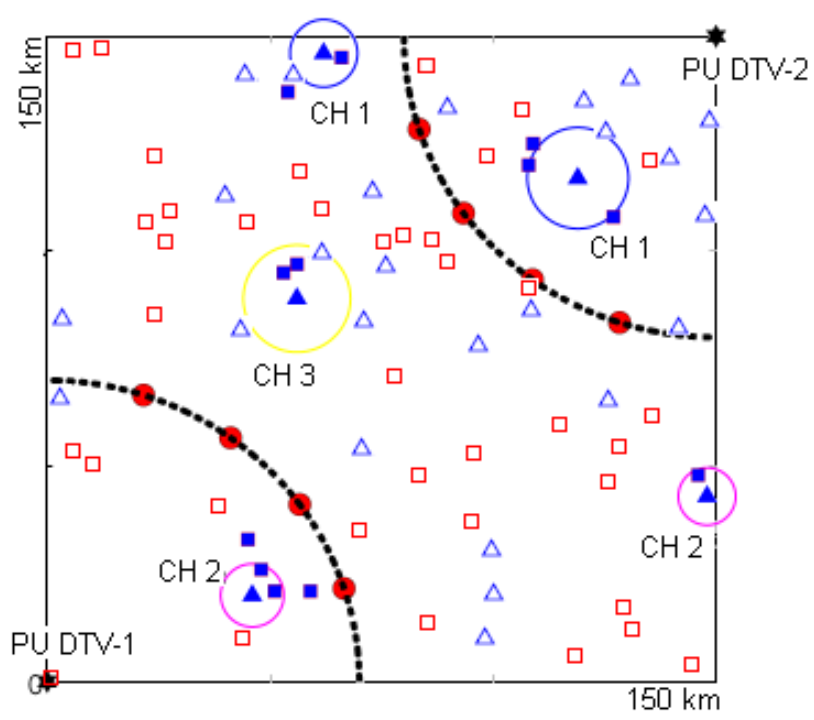

Fig. 12 Best found solution to the LP without taking into account follower's existence. PU DTV-1 operates on channel 1; PU DTV-2 operates on channels 2 and 3 


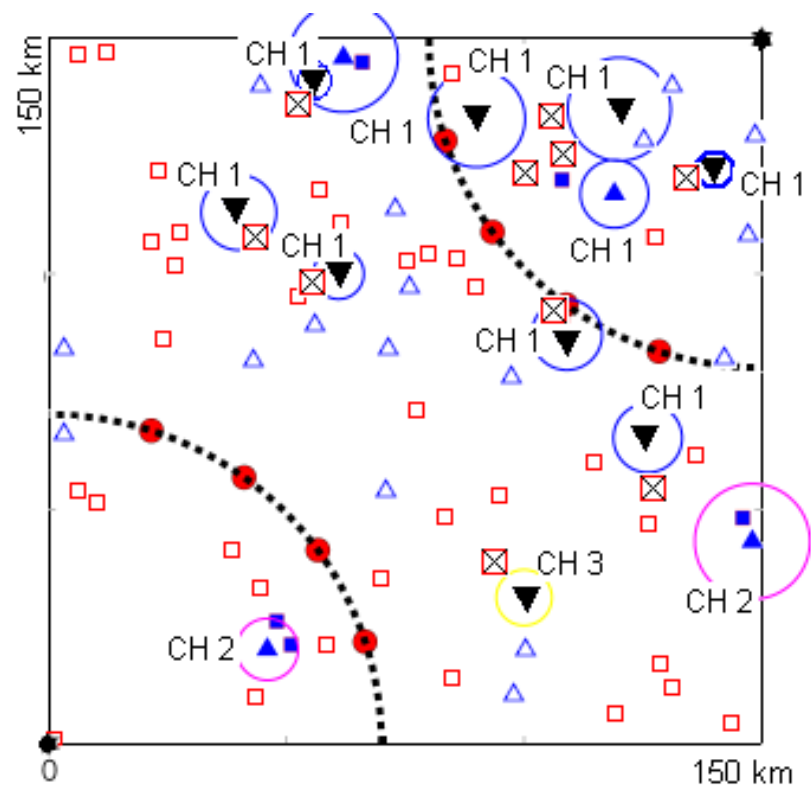

Fig. 13 Optimal reply of the cooperative-behaved follower faced with the initial leader's solution

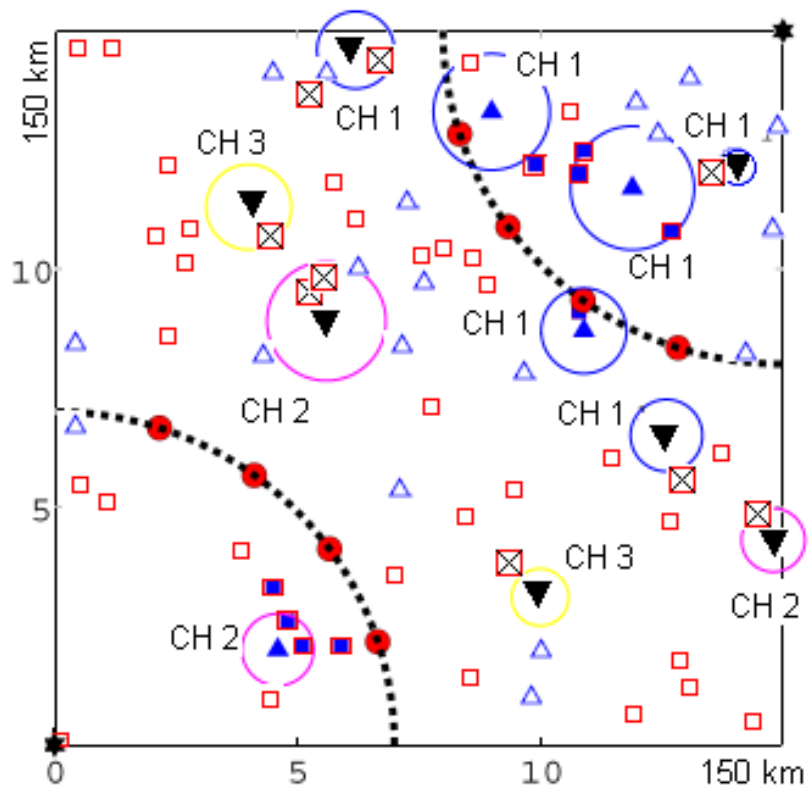

Fig. 14 Best found solution based on cooperative follower's behavior 


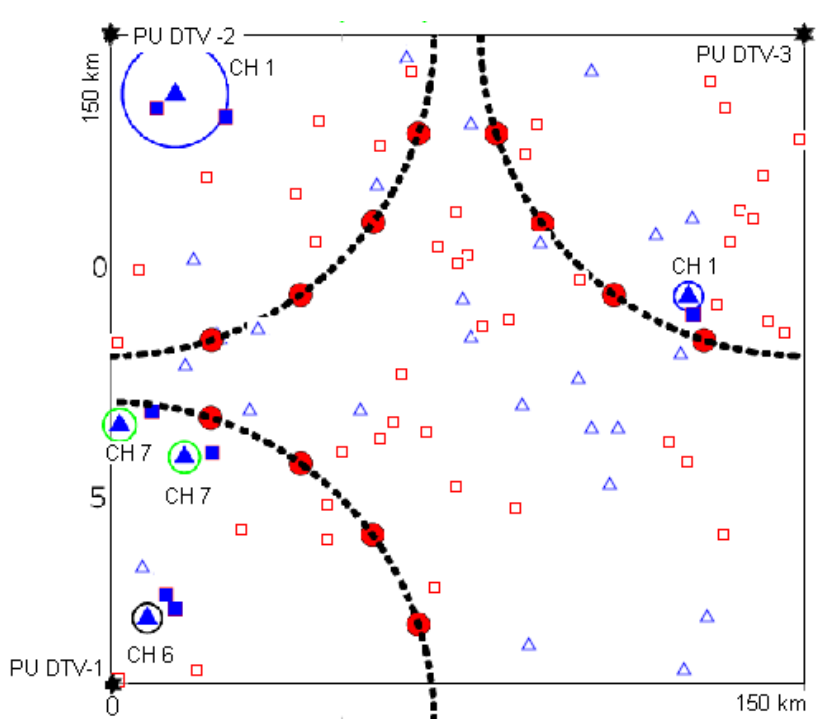

Fig. 15 Best found solution to the LP without taking into account follower's existence. There are 3 PU DTV towers

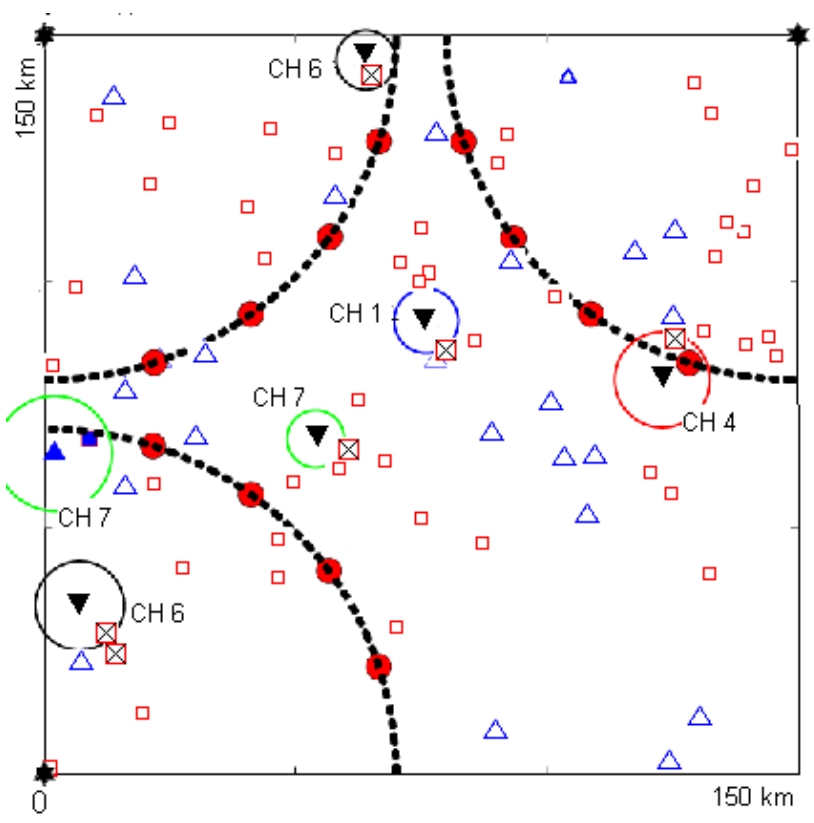

Fig. 16 Optimal reply of the cooperative-behaved follower faced with the initial leader's solution 


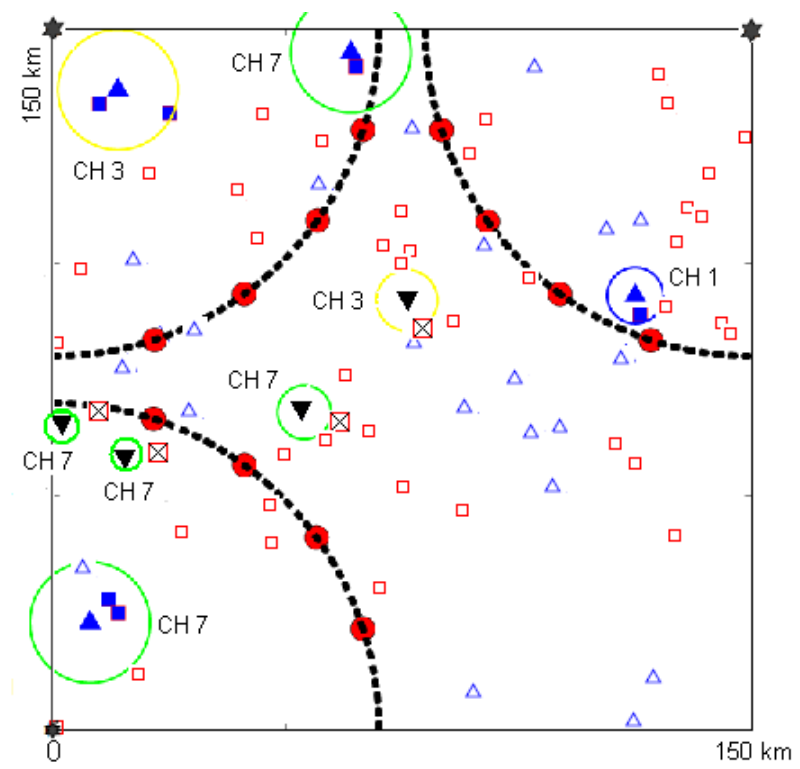

Fig. 17 Best found solution based on cooperative follower's behavior

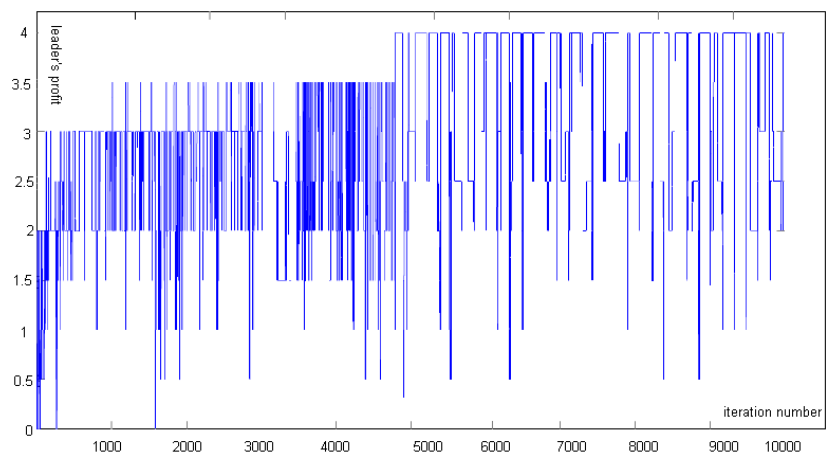

Fig. 18 Leader's profit at each iteration of the matheuristic

level mixed integer model proposed in the algorithm developed for the first time. We have established the complexity status of the problem and finding a feasible solution. Our problem is $\Sigma_{2}^{P}$-hard, and finding a feasible solution is an NP-hard in the strong sense problem. By considering cooperative and non-cooperative behaviors of the follower, we have developed a methodology to find upper and lower bounds respectively for the leader's profit. We have proposed a matheuristic based on probabilistic Tabu search and mathematical programming in order to obtain the near optimal solutions. Our numerical work shows the interest of anticipating competition in a cognitive network planning. 


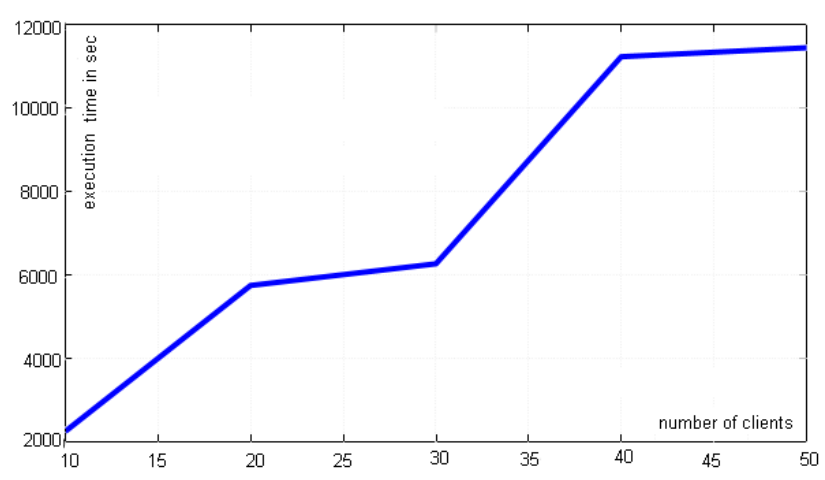

Fig. 19 Simulation execution time obtained by fixing the number of potential sites $\mathcal{S}$ to 30 and varying the number of clients

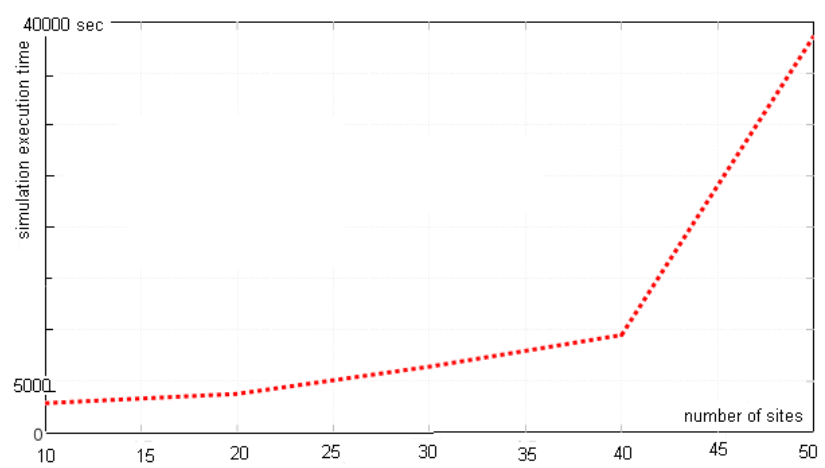

Fig. 20 Simulation execution time obtained by fixing the number of clients $\mathcal{N}$ to 40 and varying the number of potential sites

\section{References}

1. Amaldi E., Capone A., Malucelli F.: Radio Planning and Coverage Optimization of 3G Cellular Networks. Springer Wireless Networks 14(4): 435-447 (2008)

2. Alekseeva E., Kochetov Yu.: Matheuristics and exact methods for the discrete $(r \mid p)$ centroid problem. In: E-G. Talbi (Ed.), Metaheuristics for bi-level optimization. Springer, pp. 189-219 (2013)

3. Alekseeva E., Kochetova N., Kochetov Y., Plyasunov A.: Heuristic and exact methods for the discrete $(r \mid p)$-centroid problem. Evolutionary Computation in Combinatorial Optimization, ser. Lecture Notes in Computer Science. Springer 6022: 11-22 (2010)

4. Altman E., Kumar A., Singh C., Sundaresan R.: Spatial SINR Games Combining Base Station Placement and Mobile Association. Proc. of the IEEE Conference on Computer Communications (INFO-COM), Rio de Janeiro, Brazil, Apr. (2009)

5. Bard J., Moore J.: A branch and bound algorithm for the bilevel programming problem. SIAM Journal on Scientific and Statistical Computing 11(2): 281-292 (1990)

6. Beresnev V.L., Melnikov A.A.: Approximate algorithms for the competitive facility location problem. Journal of Applied and Industrial Mathematics 5(2): 180-190 (2011)

7. Boschetti M., Maniezzo V., Roffilli M., Rohler A.: Matheuristics: optimization, simulation and control. Hybrid Metaheuristics, ser. Lecture Notes in Computer Science. Springer 5818: 171-177 (2009)

8. Brewer J.: TV White Space Technology for Rural Telecommunications. Telco2, Aug. (2012) 
9. Davydov I., Kochetov Y., Plyasunov A.: On the complexity of the $(r \mid p)$ centroid problem in the plane. TOP 22(2): 614-623 (2014)

10. DeNegre S., Ralphs T. A branch-and-cut algorithm for integer bilevel linear programs. Proc. of the INFORMS Computing Society Meeting, Charleston, USA, Jan. (2009)

11. Deb S., Srinivasan V., Maheshwari R.: Dynamic spectrum access in DTV whitespaces: design rules, architecture and algorithms. Proc. of the ACM International Conference on Mobile Computing and Networking (MOBICOM), Beijing, China, Sept., pp. 1-12 (2009)

12. Gerami C.: Design methodology for backhaul and distribution networks using TV white spaces. Masters thesis, Rutgers, The State University of New Jersey, New Brunswick, New Jersey, May (2011)

13. Glover F., Laguna M.: Tabu Search. Boston: Kluwer Academic Publishers, (1997)

14. Hansen P., Jaumard B., Savard G.: New branch and bound rules for linear bilevel programming. SIAM Journal on Scientific and Statistical Computing 13(5): 1194-1247 (1992)

15. ITU-R, Method for Point-to-Area Predictions for Terrestrial Services in the Frequency Range $30 \mathrm{MHz}$ to $3000 \mathrm{MHz}$, Recommandation pp. 15-46, Sept. (2009)

16. Kim H., Shin K.: Asymmetry-aware real-time distributed joint resource allocation in IEEE 802.22 WRANs. Proc. of the IEEE International Conference on Computer Communications (INFOCOM), San Diego, USA, Mar. (2010)

17. Kochetov Y. A.: Facility location: discrete models and local search methods. In: Chvatal. V. (Ed.): Combinatorial Optimization. Methods and Applications. IOS Press, Amsterdam, pp. 97-134 (2011)

18. Kononov A.V., Kochetov Yu.A., Plyasunov A.V.: Competitive facility location models Comput. Math. and Mathem. Physics 49(6): 994-1009 (2009)

19. Kress D., Pesch E.: Sequential competitive location on networks. European J. Oper. Res. 217(3): 483-499 (2012)

20. Küçükaydin H., Aras N., Altinel I.: Competitive facility location problem with attractiveness adjustment of the follower: A bilevel programming model and its solution. European Journal of Operational Research 208(3): 206-220 (2011)

21. Legillon F., Liefoogh A., Talbi E.: A parallel metaheuristic for bilevel optimization Proc. of Metaheuristic International Conference (MIC), Udine, Italy, July (2011)

22. Liang Y.-C. and others: System description and operation principles for IEEE 802.22 WRANs. doc.: IEEE 802.22-05/0093r0 (2005)

23. Maqbool M., Coupechoux M., Godlewski P.: Comparison of various frequency reuse patterns for WiMAX Networks with adaptive beamforming. Proc. of IEEE Vehicular Technology Conference (VTC Spring), Singapore, May (2008)

24. Megiddo N., Supowit K.: On the complexity of some common geometric location problems, SIAM Journal on Computing 13(1): 182-196 (1984)

25. Mériaux F., Lasaulce S., Kieffer M.: More about base station location games, CoRR abs/1105.2243 (2011)

26. Niyato D., Hossain E., Han Z.: Dynamic spectrum access in IEEE 802.22-based cognitive wireless networks: a game theoretic model for competitive spectrum bidding and pricing. IEEE Wireless Communications 16(2) 16-23 (2009)

27. Nekovee M.: Quantifying the availability of TV white spaces for cognitive radio operation in the UK. Proc of IEEE International Conference on Communications Workshops (ICC), Dresden, Germany, June (2009)

28. Oldoni M., Macchiarella G., Seyfert F.: Synthesis and Modelling Techniques for Microwave Filters and Diplexers: Advances in Analytical Methods with Applications to Design and Tuning: Scholars' press (2014)

29. Schaefer M., Umans C.: Completeness in the polynomial-time Hierarchy: Part I: A compendium, ACM Sigact News, Complexity Theory Column 37, 33(3): 32-49 (2002)

30. Shankar N., Cordeiro C.: Analysis of aggregated interference at DTV receivers in TV bands. Proc. of the International Conference on Cognitive Radio Oriented Wireless Networks and Communications (CrownCom), Singapore, May (2008)

31. Sofer E., Segal Y., Leiba Y.: Deployment of OFDMA Based Solution in 802.22 Scenarios. Runcom, doc.: IEEE 802.22-06/0002r0 (2006)

32. Ye L., Wei Z., Du H., Xie H., Sang L., Yang D.: Accumulative interference modeling of cellular CR network to DTV system. Proc. of the IEEE Vehicular Technology Conference (VTC Fall), San Francisco, USA, Sept. (2011) 